Fortgeschrittenes Melanom

\title{
Erster PD-1-Antikörper zur Monotherapie zugelassen
}

Mit Nivolumab ${ }^{1}$ wurde im Juni 2015 der erste PD-1(Programmed death 1)-Inhibitor für die Monotherapie des fortgeschrittenen (nicht resezierbaren oder metastasierten) Melanoms bei therapienaiven und vorbehandelten Patienten zugelassen.

Der Immun-Checkpoint-Inhibitor richtet sich gegen den PD-1-Rezeptor auf der T-Zelle und verhindert so dessen Interaktion mit seinen Liganden PD-L1 und PDL2, die auf Tumorzellen hoch exprimiert sind. Die therapeutische Blockade hebt die über den PD-1-Rezeptor vermittelte Inaktivierung der T-Zelle auf, sodass das Immunsystem den Tumor wiedererkennen und zerstören kann.

Maßgeblich für die Zulassung in der Erstlinientherapie war die CheckMate066-Studie, in der 418 nicht vorbehandelte Patienten mit BRAF-Wildtyp-Melanom im Stadium IV eine Monotherapie mit Nivolumab oder eine Chemotherapie mit
Dacarbazin (DTIC) erhielten ${ }^{2}$. „Nach einem Jahr lebten in der Nivolumabgruppe noch 73\% der Patienten“, berichtete Prof. Dirk Schadendorf, Essen, auf einer Presseveranstaltung ${ }^{3}$. In der DTIC-Gruppe waren es $42 \%$. Auch das mediane progressionsfreie Überleben (PFS) war mit 5,1 Monaten vs. 2,2 Monate deutlich besser. 40\% sprachen auf die Therapie an (vs. $14 \%$ in der DTIC-Gruppe). Der Überlebensvorteil war unabhängig vom PD-L1-Expressionsstatus des Tumors. Die Studie wurde aufgrund der deutlichen Überlegenheit des PD-1-Antikörpers vorzeitig beendet und entblindet.

Die Zulassung für die Zweitlinientherapie beruht auf den Daten der CheckMate037-Studie mit 405 Melanom-Patienten, die mit Ipilimumab oder BRAF-Inhibitoren vorbehandelt waren ${ }^{4}$. Unter der Monotherapie mit Nivolumab zeigte sich eine deutlich bessere, objektive Ansprechrate von $32 \%$ gegenüber $11 \%$ in der Ver- gleichsgruppe, die eine vom Prüfarzt gewählte Chemotherapie (DTIC oder Carboplatin plus Paclitaxel) erhielt. 95\% der Patienten sprachen anhaltend auf die PD1-Blockade an; der Median wurde mit Nivolumab noch nicht erreicht, unter Chemotherapie betrug er 3,5 Monate. Das Ansprechen war wiederum unabhängig vom BRAF-Mutationsstatus und der PD-L1-Expression. Therapiebedingte Nebenwirkungen waren unter Nivolumab in den Zulassungsstudien überwiegend von Grad 1/2. Schwere unerwünschte Ereignisse Grad 3/4 waren in CheckMate-066 mit $12 \%$ vs. $18 \%$ und in CheckMate- 037 mit $9 \%$ vs. $31 \%$ unter der PD-1-Blockade seltener als in den Vergleichsarmen.

\section{Michael Koczorek, Bremen}

${ }^{1}$ Opdivo ${ }^{\circledR}$, Bristol-Myers Squibb GmbH \& Co. KGa, München

${ }^{2}$ Robert C et al. N Engl J Med 2015; 372: 320-330

${ }^{3}$ Pressekonferenz „Mit OPDIVO ${ }^{\circledR}$ von der Vision zur Wirklichkeit. Eine neue Zeit für Patienten mit metastasiertem Melanom“, 5. Europäisches PostChicago Melanoma/Skin Cancer Meeting, München, Juni 2015, Veranstalter: Bristol-Myers Squibb GmbH \& Co. KGa

${ }^{4}$ Weber JS et al. The Lancet Oncology 2015; 16: 375-384

\section{Neue Entwicklungen in der Immuntherapie}

Genmutationen sind beim Melanom besonders häufig, erläutert Prof. Axel Hauschild, Kiel, bei einem Satellitensymposium im Rahmen der DDG-Tagung in Berlin ${ }^{1}$. Etwa 30000 Mutationen seien beim Melanom beschrieben und die Tumoren, die besonders gut auf Inhibitoren oder Stimuli der Immun-Checkpoint-Blockade ansprechen, seien interessanterweise Tumoren mit sehr vielen Mutationen, ergänzt Hauschild.

Neben Ipilimumab sind mittlerweile auch Nivolumab und Pembrolizumab als Wirkstoffe in der Immuntherapie des Melanoms zugelassen. Die beiden PD-1-Antikörper Nivolumab und Pembrolizumab sind seit Ende 2014 von der Food and Drug Administration (FDA) für bereits vorbehandelte Patienten zugelassen. Studiendaten zeigen eine hohe Remissionsrate (ca. 40\%), ein gutes progressionsfreies Überleben (ca. 6 Monate) und deutliche Gesamtüberlebensvorteile gegenüber einer konventionellen Chemotherapie.
Die Zulassung der European Medicines Agency (EMA) wird nach Angaben von Hauschild Mitte dieses Jahres erwartet. Eine aktuelle Studie ${ }^{2}$ zeigte, dass die Kombination von Nivolumab und Ipilimumab wirksamer ist als Ipilimumab alleine. $\mathrm{Ob}$ diese Kombinationstherapie auch besser ist als Nivolumab alleine, blieb offen.

\section{Sicherheit der Patienten im Blick behalten}

Bei all den Daten zur Wirksamkeit der Immuntherapeutika erinnert Dr. Katharina C. Kähler, Kiel, im Rahmen ihres Vortrags daran, dass die Sicherheit der Präparate für die Patienten nicht außer Acht gelassen werden dürfe. Die häufigsten Nebenwirkungen betreffen die Haut und den Gastrointestinaltrakt, nur seltenen kommt es dabei zu schweren Fällen. Das Nebenwirkungsspektrum der CTLA-4- und der PD-1/PDL-1-Antikörper ist sehr ähnlich. Das Symptom unter dem die mit PD-1/PDL-1-Antikörpern behan- delten Patienten im Alltag besonders leiden, ist die Fatigue (36\%). Da es sich bei der Immuntherapie um eine Dauertherapie handelt, plädiert Kähler dafür, sich diesem Problem anzunehmen, um die Patienten therapietreu zu halten.

\section{Ulla Welzel, Stuttgart}

\footnotetext{
1 Satellitensymposium „Neue Entwicklungen in der Immunonkologie beim Melanom" anlässlich der 48. Tagung der Deutschen Dermatologischen Gesellschaft, April 2015, Berlin, Veranstalter: MSD Sharp \& Dohm GmbH, Haar

2 Postow MA et al. N Engl J Med 2015; 372: 2006-2017
} 